\title{
Visual Modeling Interfaces for the Cutting Stock Problem
}

\author{
L. Lessa Lorenzoni, F. J. Negreiros Gomes, \\ Universidade Federal do Espírito Santo
}

Av. Fernando Ferrari, s/n - CEP 29060-900, Vitória-ES - Brasil email: [lessa,negreiro]@inf.ufes.br

\begin{abstract}
This work describes the design and development of visual modeling interfaces for Decision Support Systems - DSS in the context of Computer Integrated Manufacturing - CIM, that are based in optimization models and algorithms. The DSS can show the model to the user and then he can form clear mental images of the structure and functions of the model. As a case study, we present an application, of Visual Interactive Modeling to the cutting stock problem in the shoe industry.
\end{abstract}

\section{Keywords}

Visual modeling interactive, Modeling by Example, Cutting Stock.

\section{INTRODUCTION}

Optimization problems arise in several fields of application. In the literature, the attention has been concentrated in the development of models and algorithms. However, concerning the input/output aspects, little effort has been presented to turn the modeling process into a comfortable task to the end user.

Specifically, in the Computer Integrated Manufacturing - CIM there is a great need of systems optimization tools, but the lack of appropriated user interfaces does not have encouraged their utilization in this area.

In this way, we have been involved in the application of visual interface concepts such as Visual Interactive Modeling - VIM, Modeling-by-Example - MbE and Objects with Dynamic Behaviour - ODB, in the design and development of intelligent interfaces for optimization problem in the context of CIM.

This paper is structured as follows. In the section 2, we present the cutting stock problem and its characterization in the shoe industry. Section 3 explains the methodology 
we use in the development of the graphical interface. Section 4 presents the design of the graphical interface to the cutting of pieces in the shoe industry.

\section{THE IRREGULAR CUTTING STOCK PROBLEM}

\subsection{Problem Characterization}

The irregular cutting stock problem, illustred by Figure 1, Provedel (1995), is settled when we desire to cut a set of moulds over a plate with the objective of minimizing the material waste involved in the cutting process.

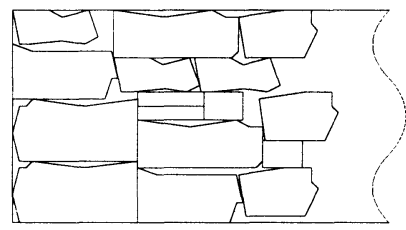

Figure 1: Cutting Stock Problem.

The pieces representation is done through circunscribed irregular polygons and the stock plate is a retangle with enough area to held the whole demand.

It should be avoided the pieces overlay, but it is allowed the rotation of the pieces over the plate. In some cases, the allocation of certain kind of pieces is allowed only into specific regions of the plate.

\subsection{The Cutting Stock Problem in the Shoe Industry}

The shoe industries use the techinical modeling approach as a manufacturing metodology. Basically, this method consists to create a shoe model or to duplicate (reproduce) the model of an existing shoe, to build the moulds, to determine the quantity of raw material to be used, to decide the moulds layout on the plate and to execute the moulds cutting process.

We note that there is a high level of raw material waste, with respect to the techinical modeling process mainly because two factors:

- it is a systematic approach;

- it uses an empirical process to decide the moulds layout.

With the objective to optimize the process of mould layout construction, Provedel (1995) presented various combinatorial models and heuristic methods.

\section{METHODOLOGY}

Aiming the systematization of the modeling process and solution of the mould allocation problem, we propose the development and implementation of a graphical visualization engine that supports the visual representation of objects as well as the active interaction 
with the user. In this way, we adopt three paradigms in the system design, which are described in the following sections.

\subsection{Visual Interactive Modeling - VIM}

Visual Interactive Modeling is the combination of friendly interactive interfaces, computer generated visual screens to represent the model state, and mathematical and symbolic problem models within Decision Support Systems - DSS, Bell (1991).

The visualization is an important characteristic in a modeling environment. It could be crucial to help a DSS user with respect to:

- to acquire new perspectives of problem structures through the generation of different views of the decision circunstances;

- to explore his own abilities in order he can recognize alternatives and significant strategies during the problem solution process.

Within a system that follows the VIM paradigm, the user of such system can increasily build, complete (fill up) and test model. This makes the man-machine interaction easier and concrete, and the modeling process stimulates the user learning concerning nonstructured situations.

\subsection{Modeling by Example - MbE}

The Modeling by Example methodology is based on interpretative-computational intelligence. It allows the decision makers to acess the knowledge, that is stored in the DSS, through a dynamic example exchange, Angehrn (1991).

The main idea that supports the interactive consulting activity is to let the system to communicate its knowledge of the problem by a concrete example exchange.

The $\mathrm{MbE}$, different from the expert systems where the main objective is to emphasize the autonomous capabilities of the system to solve problems, explores Artificial Intelligence techniques, Nilsson (1980), to improve system abilities in a high level communication with the user. The goal of the $\mathrm{MbE}$ is to give support to the decision maker creativity and intuition without attempting to substitute his judgement. It supports the user in the flexible application of complex methods into a VIM environment. So, the users do not need to interrupt their own decision process or to reformulate their models because the change from a descriptive to a normative mode.

\subsection{Objects with Dynamic Behaviour}

Taking into account that the visual aspect is a dominant characteristic of such systems, the user interface is thought as to play an important role.

In this context, depending on the type of user, the same object could have different views. We use the concept of objects with active behaviour to represent an object with various views, Camarinha-Matos and Pinheiro-Pita (1993). For example, the developed interface is configurable in accordance with the type of the user (Client, Planner, Designer and Administrator).

\section{GRAPHICAL VISULAIZATION ENGINE}

The graphical visualization engine, illustrated in Figure 2, allows the user to describe and visualize his problem in a graphical form instead of a mathematical form and it is divided 
in three modules: Model Specification, Example Exchange and Solution Visualization, which we describe bellow.

Model Specification: Allows the user to build an instance of the problem, where he specifies the plate and moulds geometry, the constraints and the parameters, by means of a visual language.

Example Exchange: In this module the user could interact dynamically with the system, evaluating the various instances of the solutions, and he can propose partial solutions and new specifications according to his own view of the problem context and experience.

Solution Visualization: Allows the model visualization and also exhibits a report with informations such as the executed algorithm, the raw material waste and processing time to generate the solution.

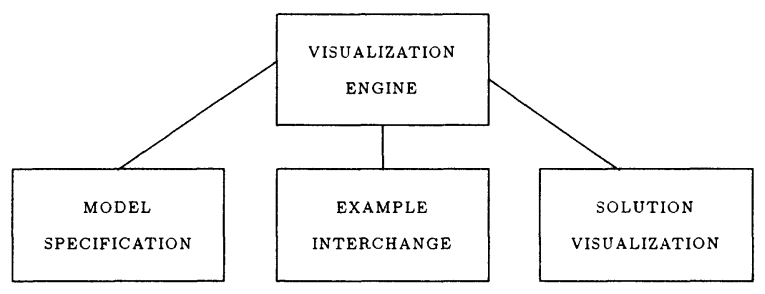

Figure 2: Graphical Visualization Engine

\subsection{Description of the Graphical Interface}

We follow the works of Alvarenga et al. (1993) and Negreiros al. (1993), where they describe respectively, the DSS project in a generic way and the user interface subsystem, in a more specific way.

The system combines the funcionality of the spreadsheets and the optimization algorithms together a graphical presentation of the results. The interface project is shown in Figure 3 .

The interface is divided in the following parts:

1. Menu Actions: exhibits the methods that can be applied to the selected object.

2. Work Area: it is reserved to the selection, interaction and presentation of problem solution.

3. Object Graphic Menu: it is composed by visual objects (icons) which could be chosen by the user to execute a desired action.

4. Status Line: it is an area reserved to the exhibition of warning, error and help messages. 


\begin{tabular}{|l|l|}
\hline 1 & \\
\hline & 3 \\
2 & \\
& \\
\hline 4 & \\
\hline
\end{tabular}

Figure 3: Graphical Interface for Cutting Stock Problem.

The interaction method is done through an Graphical User Interface - GUI that supports windows, graphic objects, mouse, menus and browsers. The interface system for the optimization models is sufficiently generic to support the model management functions, as follows:

- Model construction.

- Knowledge base interface.

- Model storage, retrieval and maintenance.

- Model and algorithms linkage.

- Model solution.

The interface configures itself in accordance to the type of user and/or in accordance to the problem to be solved. For example, in the case of the rectangular cutting stock problem, the moudls are represented by their height and width. By other side, in the case of irregular moulds, they are represented through a list of the polygon vertices that circumscribe each of them.

The DSS is constituted by three basic constructions, called modeling primitives:

- Objects: concrete or abstract entities (plate, moulds) with a set of attributes (type, area, shape, cutting direction and location).

- Relations: objects that describe functional or structural dependence among other objects (neighborhood, cutting regions).

- Constraints: restrictions on the object attribute values. For example, piece 1 must not be cut in area $\mathrm{B}$ or the permitted orientations of piece 2 .

These primitives are ready to use by user in a interactive graphic environment.

Figure 4 shows a GUI to the cutting system with respect to model building. It exhibits a screen in which the user specifies the geometry of each mould to be cut as well as various parameters and restrictions. For example, the system asks the user to input the demand for the last inserted mould (the white one in Figure 4) and the user informs that it is 4 units. 
After finishing the model building process, the user identifies it and then he is able to solve the problem, choosing the appropriated action. Figure 5 presents the solution of the proposed problem. At this point, the user can interact with the system adjusting the proposed solution in accordance to his own knowledge and experience as it is shown in the Figure 6.

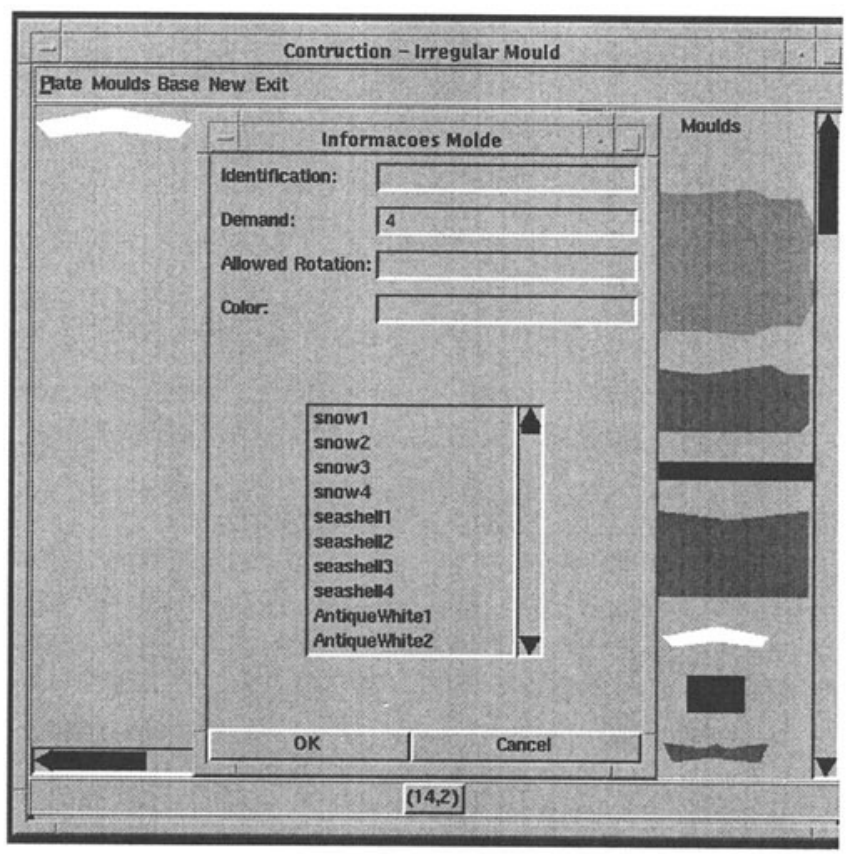

Figure 4: Model Construction - Irregular Mould. 


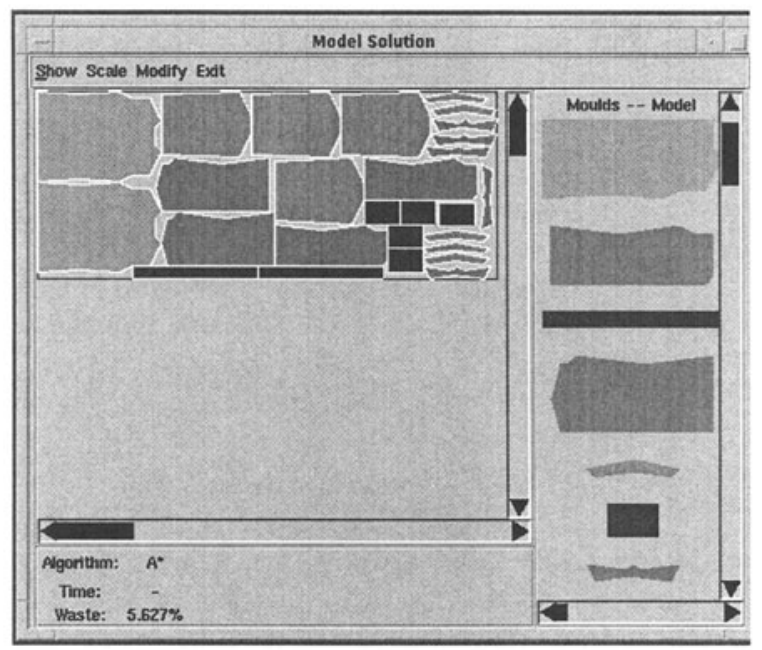

Figure 5: Solution.

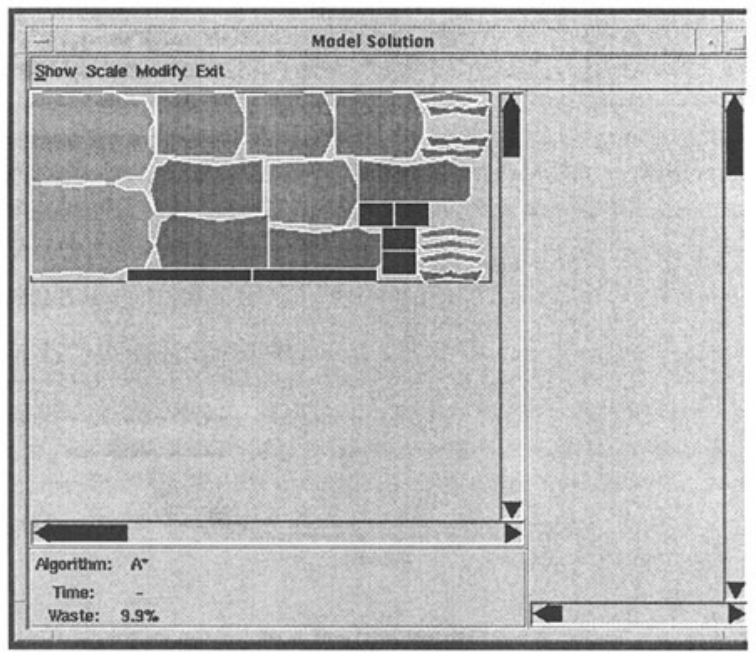

Figure 6: Modified Solution. 
A detailed description of the cutting system interface can be found in Lorenzoni (1996).

\section{CONCLUSIONS}

The implementation of the cutting system was done using an UNIX machine with the $\mathrm{X}$-Window Graphical Library together the Tk/Tcl programming language, Ousterhout (1993). The system is one of the instances of the general layout problem, Alvarenga et al. (1996), which is a common application in CIM.

The system provides the users with a modeling environment to the solution of complex problems and the man-machine interaction is done through a visual language. The interface provides an active support which allows the interaction between models and users. This is implemented through a set of active agents which autonomously fire appropriated answers, such as moulds overlay, exceeded demand, forbiden cutting regions and also supports the process of decision making. Because the system is based on active cooperation between man and computer it should be thought as a balance automation system, so it can contribute to a sustainable industrial production.

\section{REFERENCES}

Alvarenga, A.G., Provedel, A., Negreiros, F., Daza, V.P., Sastrón, F., Arnalte, S., Integration of a irregular cutting stock system into CIM. Part I - Information flows, Proceedings of the ECLA-CIM'93, Lisbon, Nov 22-26 (1993).

Alvarenga, A.G., Provedel, A., Negreiros, F., Ahonen, H., Lorenzoni, L., Daza, V.P, Pinheiro-Pita, H.J., Camarinha-Matos, L.M., Multipurpose Layout Planner for Cutting Stock Problems: Implementation Issues, Balanced Automation Systems - Architectures and Design Methods, IFIP, Chapman \& Hall, (1996).

Angehrn, A. A., Modeling by Example: A Link between Users Models and Methods in DSS, European Journal of Operational Research, Vol. 55, (1991).

Bell, P.C., Visual Interactive Modelling: the past, the present and the prospects, European Journal of Operational Reserch, Vol. 54, pp. 274-286, (1991).

Camarinha-Matos, L.M., Pinheiro-Pita, H.J., Comportamento de objectos activos na interface gráfica do sistema CIM-CASE, 4as Jornadas de PPPAC, Lisboa, (1993).

Lorenzoni, L.L., Visual interfaces for the optimization of industrial cutting in the context of CIM, MSc Dissertation, PPGEE, UFES, (1996) (in portuguese).

Negreiros, F., Alvarenga. A.G., Lorenzoni, L.L., Pinheiro-Pita, H.J., Camarinha-Matos, L.M., Objects dynamic behavior for graphical interfaces in VIM - An application case study, Proceedings of the ECLA-CIM'93, Lisbon, Nov 22-26 (1993).

Nilsson, N.J., Principles of Artificial Intelligence, Tioga Publishing Co., (1980).

Ousterhout, J.K., Tcl and Tk Toolkit, Computer Science Division, Department of Electrical Engineering and Computer Sciences, University of California, Berkeley, CA 94720, (1993).

Provedel, A., An optimization system for industrial cutting and its integration in the context of CIM., MSc Dissertation, PPGEE, UFES, (1995) (in portuguese). 